
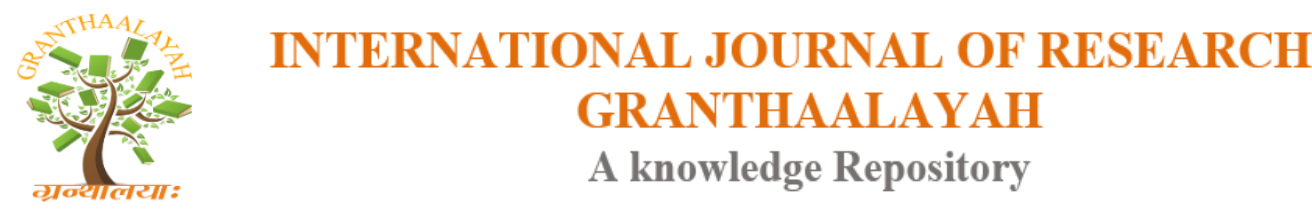

Science

\title{
HUMAN TISSUE BIOMAGNETISM ATTRACTING IRON PARTICLES AS PROPOSED MECHANISM ELUCIDATING THE IRON AND ATHEROSCLEROSIS HYPOTHESIS
}

\author{
Abraham A. Embi Bs *1 \\ ${ }^{* 1}$ Independent Citizen Scientist, 13442 SW 102 Lane Miami, Florida USA 33186
}

\begin{abstract}
The purpose of this manuscript is to introduce a mechanism that could be applied to the "Iron Atherosclerosis Hypothesis". Published data from eleven countries clearly indicate that the mortality from cardiovascular diseases is correlated with liver iron, thus the hypothesis. The bridging field of biomagnetism is herein presented as a tool for the scientific explanation for this illness. Atherosclerosis is a disease directly linked to high mortality and morbidity due to a several risks factors; and in spite of several behavioral modifications and drug therapy the prevalence of the disease continues. Amongst one of the factors influencing the disease progression is high iron levels in blood. Studies show post-menopausal women having a greater incidence of vascular disease that were correlated with higher levels of stored iron in this population. It has been stated that like any other theory in cardiovascular medicine, the iron-atherosclerosis hypothesis will undergo many trials and tribulations before it is proven or refuted. This manuscript re-introduces documentation of an intrinsic property of in vivo human tissue, which is attracting very fine iron particles. It must be noted that iron is a paramagnetic material, therefore attracted to a magnetic field.
\end{abstract}

Keywords: Iron Atherosclerosis Hypothesis; Vascular Disease; Biomagnetism Human Follicles; Imaging Sub-dermal Follicles; Biomagnetism and Iron.

Cite This Article: Abraham A. Embi Bs. (2018). "HUMAN TISSUE BIOMAGNETISM ATTRACTING IRON PARTICLES AS PROPOSED MECHANISM ELUCIDATING THE IRON AND ATHEROSCLEROSIS HYPOTHESIS." International Journal of Research - Granthaalayah, 6(8), 130-135. https://doi.org/10.29121/granthaalayah.v6.i8.2018.1406.

\section{Introduction}

The purpose of this manuscript is to introduce a mechanism that could explain the genesis of the "Iron Atherosclerosis Hypothesis". Published data from eleven countries clearly indicate that the mortality from cardiovascular diseases is correlated with liver iron, thus the hypothesis (1). Atherosclerosis is a disease directly linked to high mortality and morbidity due to a several risks factors (2); and in spite of several behavioral modifications and drug therapy the prevalence of the disease continues (3). The bridging and nascent field of biomagnetism is herein presented as a tool 
for the scientific explanation for this widespread and catastrophic illness $(4,5,6,7,8)$. Amongst one of the factors influencing the disease genesis and progression is high iron levels in blood (9). Studies show post-menopausal women having a greater incidence of vascular disease that were correlated with higher levels of stored iron in this population (10). It has been stated that like any other theory in cardiovascular medicine, the iron-atherosclerosis hypothesis will undergo many trials and tribulations before it is proven or refuted. This manuscript re-introduces documentation of an intrinsic property of in vivo human tissue attracting very fine iron particles that may open new areas of exploration or trials aimed at the elucidation of "The Iron Atherosclerosis" hypothesis. It must be noted that iron is a paramagnetic material, therefore attracted to a biomagnetic field.

\section{Materials and Methods}

Published evidence supporting the inherent electromagnetic properties of the human hair follicle will serve as a platform for the discussion section of this manuscript.

\section{Discussion}

\section{Biophysics and Biomagnetism}

Biophysics is the interdisciplinary science that applies physical principles to study biological phenomena; and it has been also described as "The Bridging Science". Biomagnetism is the branch of Biophysics where magnetic signals are recorded arising from living entities and defined as "the generation of magnetic fields by living organisms" (11).

\section{Human Tissue Biomagnetism and Iron Attraction}

A biophysical explanation is proposed as possible mechanism for the iron attraction by the arterial tissue as stated in the "Iron Hypothesis of Atherosclerosis". The aforementioned mechanism is supported by previously published research by this author and others, where using a novel optical microscopy method ex vivo and in vivo human hair follicles are shown to attract fine iron particles in solution $(4,6,7)$. Using the optical microscopy method, electromagnetic energy from hair follicles were also documented penetrating glass barriers and attracting iron particles (8). The above referenced findings were possible due to the introduction of a novel technique introduced in 2016 that has enabled via optical microscopy the documentation of magnetic fields found in living plants and animal tissue and referenced in (7).

\section{Demonstration of Human Tissue Attracting Iron Particles}

One specific image (Fig 1) shows that when hair follicles are in direct contact with Prussian Blue Stain; and crystals are void of iron particles (Panel B), there is no effect or electromagnetic attraction of crystals towards the hair follicle. Conversely, when the crystals are iron laden (Panel A) the figure shows iron laden crystals oriented (attracted) by the hair follicle electromagnetism. The definition in Physics of the magnetic attraction (orientation) of iron particles demonstrated in panel A is defined as Anisotropic. Conversely, in Panel B when the hair follicle is imbedded in a solution lacking iron particles, there is no magnetic orientation or Isotropic. 


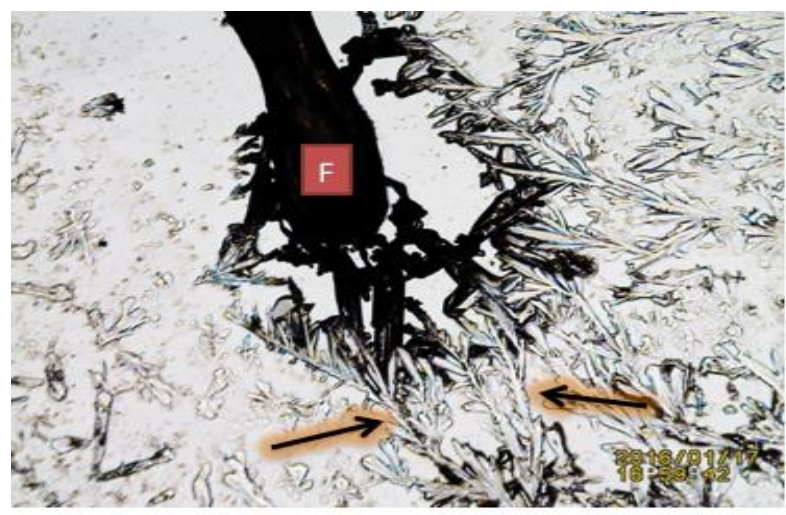

A

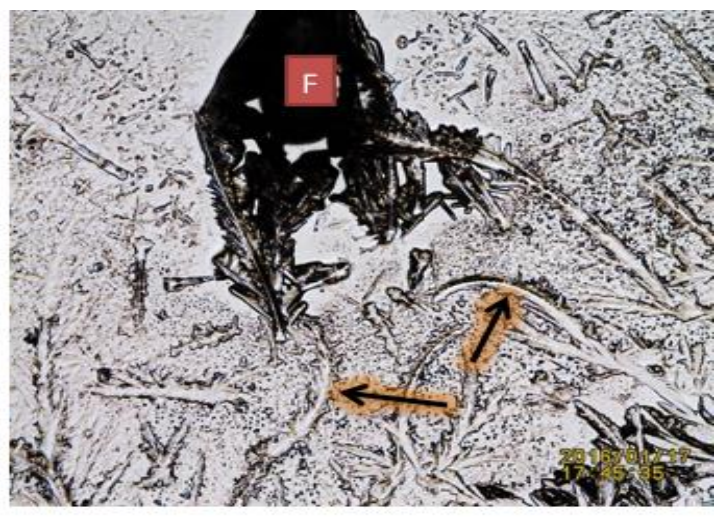

B

Figure 1: Demonstration of human hair follicle magnetism interacting with Prussian Blue Stain (no glass barrier). Panel $\mathrm{A}=$ Black arrows pointing at aligned potassium ferrocyanide crystals mixed with iron particles. Hair Follicle attracting Prussian Blue Stain + small iron particles crystals. Panel $\mathrm{B}=$ Black arrows pointing at un-aligned Potassium Ferrocyanide crystals. The hair follicle (F) biomagnetism unable to align plain Prussian Blue Stain crystals.

\section{The Sub Dermal Hair Follicle Biomagnetism Recorded in Vivo Penetrating a Glass Barrier}

Another example as shown in Fig. 2 below, is further proof of the biomagnetic attraction of hair follicles to iron particles. Panel A depicts a hair follicle outline recorded in a slide sandwich (SDW). The iron laden substance was placed in the SDW touching the skin surface. The electromagnetic fields emitted by the follicle were outlined by the iron-laden crystals in the SDW. Important to note that the biomagnetic emissions were detected remotely (outside the skin) penetrating a glass barrier. This technique and findings are described in (12). The hair follicle biomagnetic field reach was recently documented to be at $3 \mathrm{~mm}$ vertical distance (13), thus adding credence to the images.

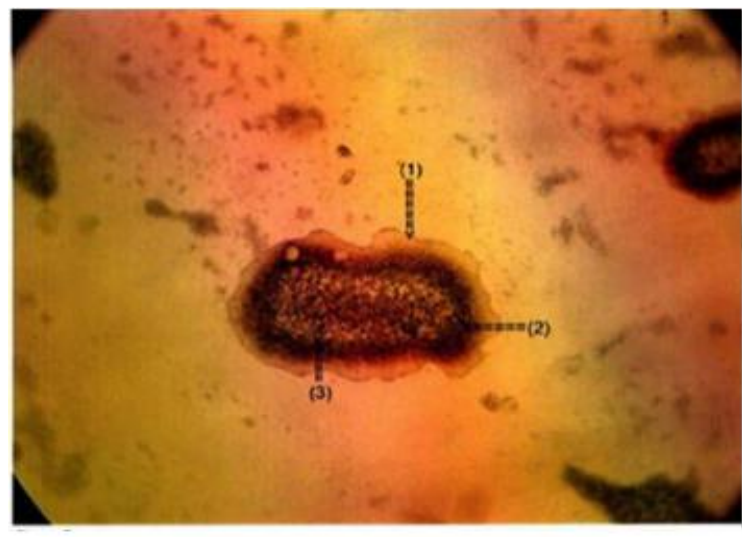

A

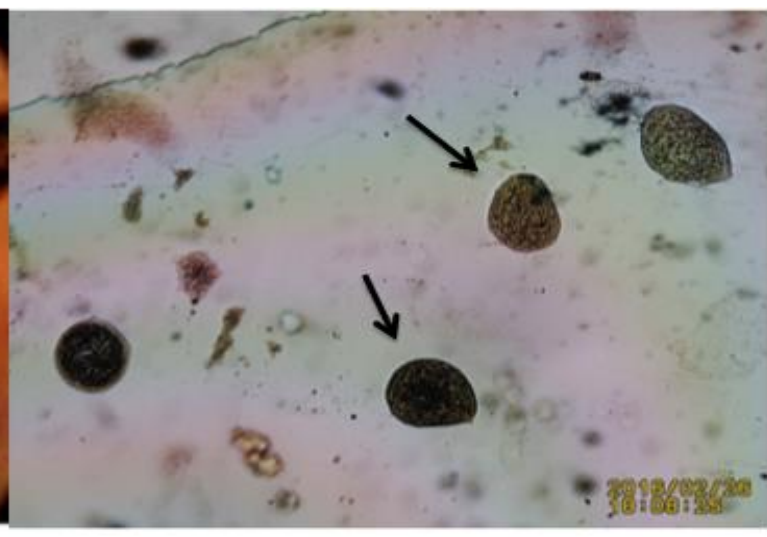

B

Figure 2: Panel A depicts a picture of a sub dermal human hair follicle virtual image, detected and published by Dr Benjamin Scherlag from The University of Oklahoma in (Scherlag BJ, Sahoo K (2016) Electromagnetic Imaging of Subdermal Human Hair Follicles In Vivo. Journal of Nature and Science (JNSCI), 2(2): e174.) 
Panel B Also depicting (black arrows) several unpublished virtual sub dermal human hair follicles obtained by this author; thus corroborating Dr Scherlag's 2016 published findings.

\section{Additional Evidence of Human Tissue Biomagnetism}

\section{Ancillary Video-Recordings}

The reader is encouraged to click the links below to view published video-recordings where a hair follicle is seen interacting (through a $1 \mathrm{~mm}$ glass barrier) with iron-laden Prussian Blue Solution. In this instance, the plucked hair follicle was sandwiched between two $25 \times 75 \times 1 \mathrm{~mm}$ glass slides. On the top slide drops of Prussian Blue mixed with iron particles were allowed to evaporate. The video-recordings show the human tissue interacting with iron laden solutions.

Https://Www.Youtube.Com/Watch?V=8pipwjvytrk\&T=30s

The same experiment was done, this time also with crystals laden diamagnetic Prussian Blue Stain. Notice the rapid advance of the ferrocyanide evaporation line during the first 29 seconds. As the evaporation line reaches the hair follice/shaft outline, there is a noticeable delay in the rate of evaporation progression. This is attributed to the biomagnetic interaction of the hair follicle with the iron laden crystals. This is an example of repulsion caused by biomagnetism of a human hair follicle being detected by iron particles through a $1 \mathrm{~mm}$ glass barrier. See video-link below.

https://youtu.be/6iKcEvxY_zE

These next video-recordings showed the first time in 2105 where the hair follicles intrinsic electromagnetism is seen interacting (attracting) iron particles as published by this author in $(6,13)$.

http://www.jnsci.org/files/html/e55.htm

\section{Intracoronary Blood Flow and Biomagnetism}

The circulatory system consists of arteries and veins. The arteries bend and twist as required by the anatomical architecture. Could this change in blood flow dynamics trigger biomagnetic fields?

I will leave you with a drawing reproduced with permission from the Journal's editor.

Drawings below reproduced from Zegers ES, Meursing BTJ, Zegers EB, Oude Ophuis AJM. Coronary tortuosity: a long and winding road. Netherlands Heart Journal. 2007;15(5):191-195. 


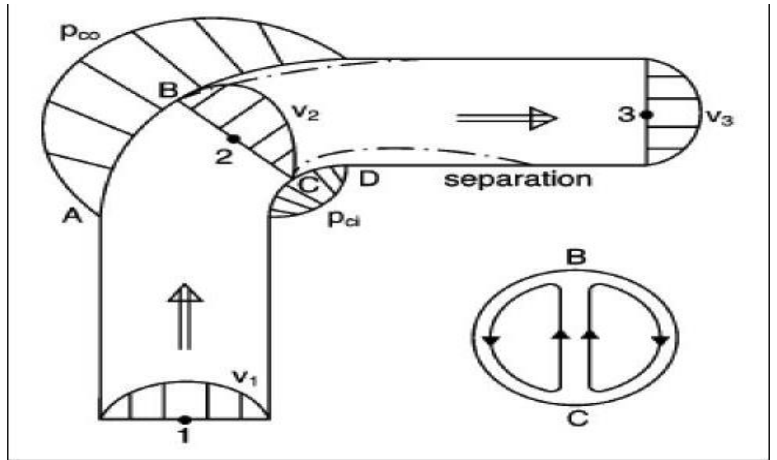

Drawing mimicking a bending human artery and the energy exchanges caused by friction. Eddies are created.

\section{Bends give extra energy loss, almost entirely caused by eddies, which originate because the flow has to separate from the wall due to a sharp bend (separation).}

\section{Summary}

The data hereby presented is in support of the "Iron and Atherosclerosis Hypothesis". In vivo and ex vivo experiments on human tissue (hair follicles) have been shown to interact (attract) iron particles via an electromagnetic principle. This phenomenon is demonstrated in several experiments by different authors and venues. Ancillary video-recordings are shown to help the reader visualize the human tissue attracting iron particles.

\section{Blood Arterial Flow as Bioelectromagnetical Inductor}

The question arises:

Q: Do the blood flow dynamics in the arterial tree generate biomagnetic fields capable of attracting the serum ferritin (read iron)?

A: Yes, the significance of turbulence in hemic systems has been published to correlate with the distribution of atheroscherotic lesions; and (Fluid turbulence Eddy) is known to induce magnetic fields $(14,15)$.

The documentation herein presented supports a bioelectromagnetic mechanism supporting "The Iron and Atherosclerosis Hypothesis".

\section{References}

[1] Yuan XM, Li W. The iron hypothesis of atherosclerosis and its clinical impact. Ann Med. 2003;35(8):578-91.

[2] 2)Daniel G. Hackam, MD; Sonia S. Anand, MD, PhD, FRCP Emerging Risk Factors for Atherosclerotic Vascular Disease: A Critical Review of the Evidence JAMA 2003;290(7):932-940. doi:10.1001/jama.290.7.932

[3] Marcio Sommer Bittencourt and Rodrigo Julio Cerci. Statin effects on atherosclerosis plaques: regression or healing? BMC Med; 2015 13:260. Doi: 10.1186/s12916-015-0499-9.

[4] Scherlag BJ, Sahoo K, Embi AA. Novel and Simplified Method for Imaging the Electromagnetic Energy in Plant and Animal Tissue. Journal of Nanoscience and Nanoengineering. $2016 \mathrm{Vol} 2$ No 1, pp 6-9. 
[5] Jerry Jacobson, Benjamin J. Scherlag. Aging and magnetism: Presenting a possible new holistic paradigm for ameliorating the aging process and the effects thereof, through externally applied physiologic PicoTesla magnetic fields. Medical Hypotheses 2015 Volume 85, Issue 3, Pages 276286

[6] Abraham A. Embi, Jerry I. Jacobson, Kaustuv Sahoo, Benjamin J. Scherlag. Demonstration of Inherent Electromagnetic Energy Emanating from Isolated Human Hairs.2015 JNSCI, Vol 1 No. 3 e55

[7] Scherlag BJ, Sahoo K. Electromagnetic Imaging of Subdermal Human Hair Follicles In Vivo. Journal of Nature and Science (JNSCI) 2016, 2(2):e17

[8] Embi, AA, Scherlag BJ. Demonstration of Human Hair Follicle Biomagnetic Penetration Through Glass Barriers. International Journal of materials Chemistry and Physics. 2016. Vol 2, No 2, 71 74. doi:10.1016/0360-1323(92)90037-P

[9] Moacir Fernandes de Godoy; Isabela Thomaz Takakura; Rafael Dinardi Machado; Luciano Vaccari Grassi; Paulo Roberto Nogueira. Serum ferritin and obstructive coronary artery disease: angiographic correlation Arq. Bras. Cardiol. 2007 vol. 88 no.4 São Paulo. http://dx.doi.org/10.1590/S0066-782X2007000400011

[10] Sullivan JL. Iron and the sex difference in heart disease risk. 1Lancet.1981 1(8233):1293-4.

[11] Baule G.M. Detection of the magnetic field of the heart. American Heart Journal 1963;66-95-6

[12] Scherlag BJ, Sahoo K Electromagnetic Imaging of Subdermal Human Hair Follicles In Vivo. Journal of Nature and Science (JNSCI), 2016 2(2): e174.

[13] Embi AA. The Human hair Follicle Pulsating Biomagnetic Field Reach as Measured by Crystals Accretion. International Journal of Research Granthaalayah. 2018 vol 6 (Iss.7). DOI: 10.5281/zenodo.xxxxxx.

[14] The significance of turbulence in hemic systems and in the distribution of the atherosclerotic lesion Wesolowski, Sigmund A. et al. Surgery, Volume 57, Issue 1, 155 - 162

[15] Texon M, Imparato AM, Helpern M. The Role of Vascular Dynamics in the Development of Atherosclerosis. JAMA. 1965;194(11):1226-1230. doi:10.1001/jama.1965.03090240060016

\footnotetext{
*Corresponding author.

E-mail address: embi21@att.net
} 